\title{
A Survey on Node Clustering in Cognitive Radio Wireless Sensor Networks
}

\author{
Gyanendra Prasad Joshi and Sung Won Kim * \\ Department of Information and Communication Engineering, Yeungnam University, 280 Daehak-Ro, \\ Gyeongsan, Geongbuk 38541, Korea; joshi@ynu.ac.kr \\ * Correspondence: swon@yu.ac.kr; Tel.: +82-53-810-2483 \\ Academic Editor: Kemal Akkaya \\ Received: 13 June 2016; Accepted: 6 September 2016; Published: 10 September 2016
}

\begin{abstract}
Cognitive radio wireless sensor networks (CR-WSNs) have attracted a great deal of attention recently due to the emerging spectrum scarcity issue. This work attempts to provide a detailed analysis of the role of node clustering in CR-WSNs. We outline the objectives, requirements, and advantages of node clustering in CR-WSNs. We describe how a CR-WSN with node clustering differs from conventional wireless sensor networks, and we discuss its characteristics, architecture, and topologies. We survey the existing clustering algorithms and compare their objectives and features. We suggest how clustering issues and challenges can be handled.
\end{abstract}

Keywords: node clustering; cognitive radio; cognitive radio wireless sensor networks

\section{Introduction}

Communications in wireless sensor networks (WSNs) is event-driven (i.e., whenever an event is triggered, wireless sensor (WS) nodes generate bursty traffic). Conventionally, WSNs are designed to work on a single channel with non-rechargeable/irreplaceable batteries and a very low active duty cycle. In a dense network environment, WS nodes deployed in the same area might try to access a channel whenever a new event triggers communications, which results in scarce bandwidth to send data packets from all the nodes in the region. These days, an increasing number of sensitive and critical activities are being monitored and observed using bandwidth-hungry multimedia WSNs. Cognitive techniques are now being used in wireless sensors to circumvent the bandwidth limitations imposed by conventional WSNs. Integration of cognitive devices in WS nodes extends their capability to utilize unused spectrum and provides greater bandwidth. The cognitive radio wireless sensor network (CR-WSN) is a candidate for the next generation of WSN systems [1-4].

Wireless sensors are normally deployed in inaccessible terrain. Therefore, the self-configuring, self-organizing abilities and the lifetimes of the WS nodes have become very important. Along with these capabilities in sensor nodes, the following are the basic characteristics of cognitive radio wireless sensor (CR-WS) nodes.

- Nodes can work with multiple channels.

- Nodes listen and follow instructions from the base station.

- They can switch channels within a pre-specified time.

- They broadcast/unicast spectrum information to neighbors.

- They feature spectrum sensing, analyzing, predicting, decision making, and management.

Details about CR-WSNs were outlined by Joshi et al. [1]. In this paper, we use the terms spectrum and channel, and node clustering and clustering interchangeably.

Most of the WSNs that measure physical phenomena, like temperature, humidity, location and movement of objects, etc., are delay-tolerant and require low bandwidth. Therefore, sensors with CR 
capabilities are basically not required. CR-WSNs are generally required for monitoring systems where low delay, high throughput, and reliability are essential. These requirements are especially applied to multimedia applications.

Logically grouping and consolidating similar sensor nodes in their proximity with certain objectives is called node clustering. A clustered wireless sensor networking architecture is advantageous to a non-cluster-based architecture in various ways. A non-cluster-based architecture is also called a single-tier network architecture and is based on a flat topology (discussed in Section 3). Node clustering enables bandwidth reuse and efficient resource allocation; thus, it can improve system capacity. Particularly, in a large scale and a dense sensor network, a single-tier network can overload the gateway node, leading to congestion and delay in communications. The single-tier network is not scalable for a larger set of sensors deployed over a large area.

Clustering in CR-WSNs is still in its infancy. There has been plenty of work done in clustering for mobile ad hoc networks (MANET) [5,6], WSNs [7], and cognitive radio networks (CRNs) [8,9]. Although some clustering issues have been addressed in the literature [10-28], clustering still remains a vastly unexplored domain in CR-WSNs.

This work attempts to explore the requirements of node clustering in CR-WSNs, compares node clustering in CR-WSNs and WSNs, and discusses the issues and challenges of node clustering in CR-WSNs. The goals of this work are as follows: (1) to make a large audience aware of the requirements of clustering schemes in CR-WSNs; (2) to provide information about the differences and similarities between clustering in WSNs and CR-WSNs; (3) to encourage researchers to propose suitable clustering algorithms for CR-WSNs, because this is a new research area, and is still in its infancy; (4) to highlight a few strengths and weaknesses of the existing algorithms; and (5) to provide a comprehensive analysis of the clustering mechanism and encourage proposals for the best strategy.

In this paper, the challenges and essence of clustering in CR-WSNs are described and discussed. Clustering in conventional WSNs is described in Section 2. Characteristics, architectures, and topologies are explained in Section 3. Objectives and advantages of node clustering in CR-WSNs are discussed in Section 4. Comparison of clustering in CR-WSNs and WSNs is presented in Section 5. Section 6 surveys the existing literature. Issues and challenges are detailed in Section 7. Finally, Section 8 concludes the paper.

\section{Clustering in Conventional WSNs}

Because wireless sensor nodes are battery-operated tiny devices, energy conservation has significant importance when designing the physical layer for WSNs. They are deployed in harsh environmental conditions, and the sensor devices are inaccessible after deployment. Therefore, it is not always possible to replace the sensors' batteries. Valid, accurate, and reliable data collection basically depends on the sensors' lifetime, which is determined by residual energy of the system. Hence, the most important challenge in WSNs is the efficient use of energy resources.

In WSNs, energy consumed for sensing and computation is much lower than for communications [29]. Therefore, a majority of the total energy consumed in WSNs is transmission power, and the required transmission power grows exponentially with an increase in transmission distance. In general, the minimum output power required to transmit a signal over distance $d$ is proportional to $d^{n}$, where $2 \leq n<4$. The exponent $n$ is closer to 4 for low-lying antennae and near-ground channels, as is typical in wireless sensor network communications [30]. Therefore, routes that have more hops with shorter hop distances can be more power-efficient than those with fewer hops but longer hop distances. Clustering encourages sensor nodes to communicate in multihop fashion, which extends the lifetime of WS networks. If the node density is high enough, it can also effectively overcome shadowing and path loss effects.

There are several strategies in the literature to conserve energy and extend WSN lifetime, such as data reduction [31], protocol overhead reduction [7], reducing the duty cycle [32], energy-efficient routing design [33], energy-efficient MAC design [10,34], and energy harvesting [35,36]. These strategies 
reduce energy consumption by controlling and mitigating the sensing process, the communications process, collisions, overhearing, control packets, idle listening, and interference [7,31-34]. Among these strategies, the ability to harvest energy from the environment is highly dependent on many environmental factors. The current state of technology in energy harvesting is still unable to provide a sustained energy supply to enable WSNs to operate continuously $[35,36]$.

Clustering has the advantages of load balancing and scalability, and can extend WSN lifetime when the network size grows. In clustering, wireless sensor nodes are usually grouped into physically disjointed, and often non-overlapped, clusters. All the contiguous nodes are in one or more clusters according to a cluster formation algorithm. Based on the clustering schemes, there is a leader in each cluster, often called a cluster head $(\mathrm{CH})$. The $\mathrm{CH}$ is responsible for data aggregation, information dissemination, and network management, and the cluster members (CMs), i.e. non-cluster head nodes, are responsible for event sensing and information collection from their surroundings.

$\mathrm{CHs}$ perform the task of intra- and inter-cluster management and data processing. Some of the responsibilities of $\mathrm{CHs}$ include coordination between inter-clusters and intra-clusters, such as cluster formation, data aggregation, and communications with the base station (BS). Each WSN consists of one or more sinks or base stations collecting information from sensor nodes. Figure 1 shows a conventional clustering structure of a typical WSN. As shown in Figure 1, sensor nodes are divided into a number of virtual groups (dotted lines) based on predefined clustering schemes. Inside a cluster, a sensor node may have a different status or function, such as a $\mathrm{CH}$, or a $\mathrm{CM}$. Several types of clustering exist, such as static, dynamic, single-hop and multihop, homogeneous and heterogeneous.

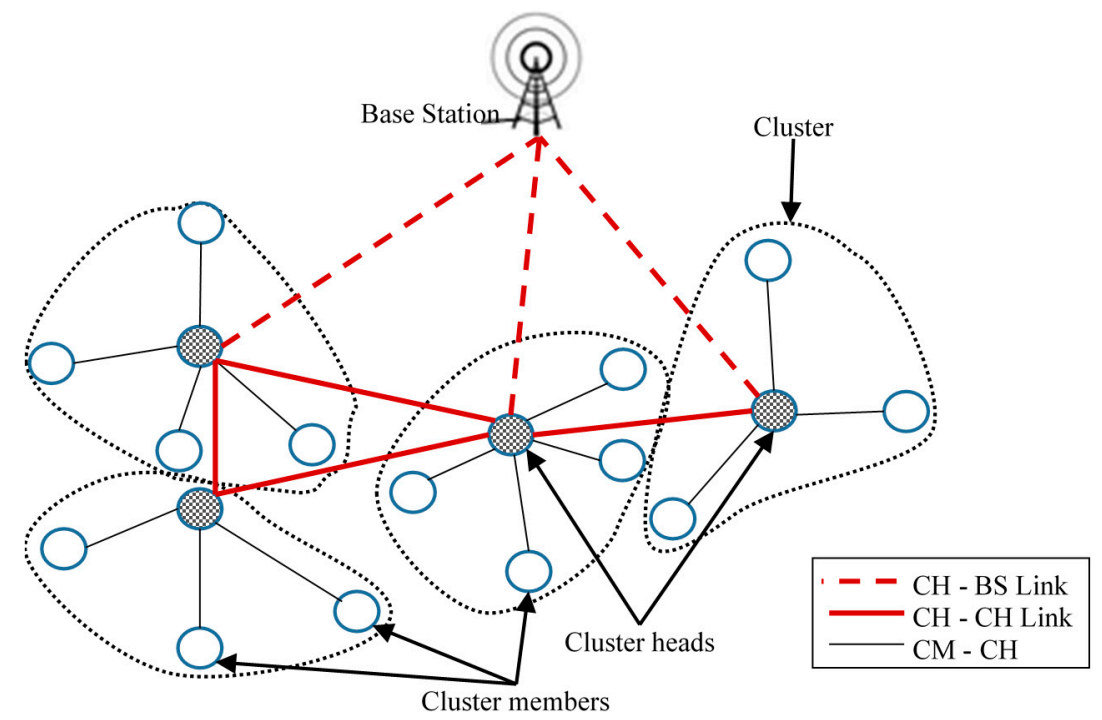

Figure 1. Conventional typical WSN cluster structure.

\section{CR-WSN Characteristics and Architecture}

\subsection{Characteristics of $C R-W S N s$}

The existing clustering algorithms in the literature [11-28] consider homogeneous CR-WS nodes, and $\mathrm{CHs}$ are selected iteratively based on residual energy and other parameters. Most of these clustering methods are similar to conventional clustering for WSNs, and seldom consider the complexities of CR-WSNs.

In CR-WSNs, the $\mathrm{CH}$ should continuously monitor the channel status, i.e. whether the channel is occupied by any incumbent users, also called primary users (PUs). The $\mathrm{CH}$ receives spectrum information from the BS and neighbor nodes in its vicinity, and negotiates and allocates available channels. Therefore, the $\mathrm{CH}$ requires dedicated hardware, and conventional methods of $\mathrm{CH}$ selection 
that are applied in WSNs (based on geographic region, load, residual energy, links, etc.) do not provide enough parameters. Nevertheless, the methods applied by the conventional $\mathrm{CH}$ selection algorithms could be considered when choosing an ideal place to deploy a cluster head (where it can cover the maximum number of neighbor sensor nodes) or when determining the shortest distance from the base station, among other things.

Resources in WSNs are greatly constrained in terms of power supply, processing capability, and transmission bandwidth. Different from WSNs, a CH in a CR-WSN may need to be powered by a heavy-duty battery. Additionally, processing capability, memory, etc., should be enhanced significantly.

It is better to design a global addressing scheme, such as Internet Protocol (IP) for better protection of the license users' right to access the incumbent channels. With a global addressing scheme, it is easy for the BS to know which wireless sensor node is using which particular channel in certain geographical location. This information helps BS to allocate a channel that has less probability of PUs' arrival, which mitigates the collision probability with the PUs and protects the right of PUs to access the channel. Applying IP in WSNs is difficult, because address updating in a large-scale or dynamic WSN can result in heavy overhead. However, CR-WSNs can be designed with IP addresses, because the $\mathrm{CH}$ has special hardware. Table 1 summarizes the differences between clustering in WSNs and CR-WSNs.

Table 1. Clustering differences between WSNs and CR-WSNs.

\begin{tabular}{ccc}
\hline Factors & WSNs & CR-WSNs \\
\hline Channels & $\begin{array}{c}\text { Usually single channel } \\
\text { (multiple channels possible) }\end{array}$ & Multiple channels are required \\
\hline $\begin{array}{c}\text { Protection of } \\
\text { PU access priority }\end{array}$ & Not an issue & $\begin{array}{c}\text { Must be protected; incumbent license } \\
\text { holder for the channels must get priority }\end{array}$ \\
\hline Hardware & Readily available (off-the-shelf) & Not readily available; still at the R\&D level \\
\hline Sensor form factor & Small & Moderate to large \\
\hline Memory & Limited & High \\
\hline CH & Any node can take a CH role & Mostly dedicated; role changes if all WS \\
nodes have CR
\end{tabular}

\subsection{Types of CR-WSNs}

Based on the types of nodes deployed, CR-WSNs can be categorized into two kinds.

\subsubsection{All WS Nodes Equipped with CR (CR-WSN-all)}

In this type of CR-WSN, all WS nodes are equipped with a CR module. These CR-WS nodes, also called secondary users (SUs), in the network sense available spectrum within a particular region and report to the base station (BS) or an intermediate node. The existing clustering algorithms in 
the literature consider this type of CR-WSN. Node deployment and clustering is easy in this type of network. However, it is costlier in terms of form factor and energy consumption.

\subsubsection{Few Nodes with CR Ability (CR-WSN-few)}

In this type of network, only few nodes have a CR module, and other nodes have only limited $\mathrm{CR}$ or no CR module. If the performance of a CR-WSN-all and a CR-WSN-few is similar, embedding a CR module in each sensor with higher hardware and energy costs is unnecessary. CR-WSNs in which only $\mathrm{CHs}$ are equipped with $\mathrm{CR}$ for local spectrum sensing and channel assignments could be a better approach.

In CR-WSN-few, nodes with a CR module work as a coordinator node (a cluster head after cluster formation). They sense the local spectrum and report to the BS. That node broadcasts the channel selection decision to other neighbor nodes. This type of network is advantageous in terms of cost and energy consumption. However, coordinator node deployment is difficult, and may create a single point of failure.

\subsection{Node Deployment}

Deployment of CR-WS nodes directly affects the network-setup cost and spectrum information-gathering capability of the CR-WSN. Node deployment is a critical task, as it should be optimal in order to increase network lifetime, PU protection, and cost-effectiveness. The deployment cost depends upon the number of WS nodes with CR that are deployed in the particular area. Basically, CR-WS nodes can be deployed in two ways: deterministic and non-deterministic.

\subsubsection{Deterministic Deployment}

This is also called structured deployment, or regular deployment. This type of deployment is done in an indoor environment or in a small and favorable environment. All types of CR-WSNs (see Section 3.2) can be considered for this type of deployment, which provides the best possible coverage, and allows easier clustering. Common types of deterministic deployment strategies are the triangular grid, the rectangular grid, and the hexagonal grid. The coverage area of each node in triangular grids, rectangular grids, and hexagonal grids are $(\sqrt{3} / 4) R^{2}, R^{2}$, and $(3 \sqrt{3} / 4) R^{2}$, respectively, where $\mathrm{R}$ is the distance between any adjacent nodes. The total number of nodes required to cover a given area, A, in a triangular grid is $\left\lceil\frac{4 A}{\sqrt{3} R^{2}}\right\rceil$, in a rectangular grid, is $\left\lceil\frac{A}{R^{2}}\right\rceil$, and in a hexagonal grid, is $\left\lceil\frac{4 A}{3 \sqrt{3} R^{2}}\right\rceil$. Deterministic deployment is recommended for a fixed location where fewer sensors are deployed. It is easy to maintain, and has lower maintenance costs. For uninterrupted performance, multiple $\mathrm{CHs}$ should be deployed.

\subsubsection{Nondeterministic Deployment}

This is also called unstructured deployment or random deployment. Generally, sensor networks are targeted at inaccessible, unattended, and possibly hostile environments. Therefore, sensor nodes are deployed by relatively uncontrolled means, such as being dropped by a helicopter, or released from an airplane. Examples of such deployment models are uniform distribution and Poisson distribution. Assuming that $N$ WS nodes are uniformly distributed over area $A$, the WS node density can be given by $\lambda=N / A$. In a Poisson distribution, the probability that there are $m$ WS nodes within area $S$ is $P(m)=\frac{(\lambda S)^{m}}{m !} e^{-\lambda S}$ [37]. This type of deployment is recommended for non-reachable places, such as deep forests, within the sea, in harsh deserts, on mountains, or in remote areas. A CR-WSN-all (see Section 3.2) is recommended for this type of network, because deployment of a $\mathrm{CH}$ that covers all the nodes in the network is difficult to achieve.

With the CR-WSN-few type, multiple $\mathrm{CH}$-capable nodes should be deployed for the $\mathrm{CH}$ and backup $\mathrm{CH}$ nodes. 


\subsection{Classification of CR-WSNs According to Network Topology}

The two basic types of topology used for dissemination and gathering are the flat topology and the hierarchical topology.

\subsubsection{Flat Topology}

In this type of network, the BS directly aggregates data using routing algorithms. The BS broadcasts channel availability information to its members using a common control channel (CCC), and the CR-WS nodes hop to the channel and communicate with the BS via multihop routes. Based on the types of node deployed, the following two types of flat topologies can be considered for CR-WSNs.

\section{(a) Flat CR-WSN with All CR-WS Nodes (Flat CR-WSN-all)}

This is similar to traditional WSNs, where the BS usually broadcasts a query message, and the WS nodes that have data matching the query will send a response to the BS. The BS updates spectrum availability information (SAI) from the spectrum database for the particular geographic region that is maintained by the authority providing the local or central spectrum information. The BS finds the common channels between the SAI and its own sensing result, and then broadcasts a channel availability message to the CR-WS nodes in its vicinity. The CR-WS nodes again compare the channel list received from the BS to their own channel-sensing results and select the best channel to communicate with the BS.

Figure 2 shows the flat network topology. The WS nodes usually communicate in multihop fashion by using peer nodes as relays. This network is very expensive in terms of deployment cost and energy efficiency. This type of network has a scalability issue, and suffers from the energy hole problem. Therefore, it is recommended for a small geographic region.

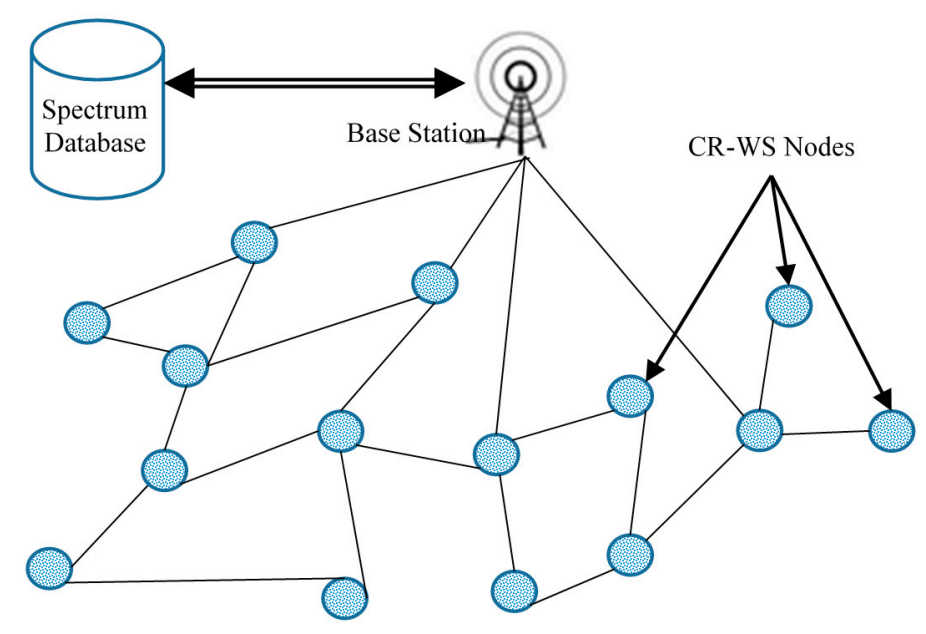

Figure 2. Flat network topology with all CR-WS nodes.

\section{(b) Flat CR-WSN with Only Few CR-WS Nodes (Flat CR-WSN-few)}

This is similar to a Flat CR-WSN-all network; however, only few nodes have CR module, unlike the previous category. All nodes do not participate in the channel-sensing and channel-selection processes. Only the nodes with CR module sense channels and send available channel information to the BS, and all other nodes just follow the BS's broadcast message for channel selection and channel switching. In this network, "node with CR module" deployment with proportional representation for all targeted geographical reason is difficult. However, overall hardware cost is comparatively inexpensive, because not all WS nodes require CR modules. 


\subsubsection{Hierarchical Topology}

Here, data aggregation is accomplished by structuring CR-WS nodes into clusters. A node that is responsible for data aggregation and fusion is called a cluster head. Data aggregation and fusion at the $\mathrm{CH}$ reduces the number of messages transmitted to the $\mathrm{BS}$, and hence, mitigates energy consumption and conserves bandwidth by reducing the number of nodes taking part in transmissions.

Conventionally in WSNs, clustering is based on several parameters, such as node deployment, cluster count, inter-cluster communications, node and $\mathrm{CH}$ mobility, node types, node roles, node identifiers, node connectivity, neighborhood information, the cluster formation algorithm, the power conservation method, $\mathrm{CH}$ roles, and the $\mathrm{CH}$ selection method. The classification of clustering methods in WSNs has been well studied [32,38-44].

In addition to the above-mentioned parameters, clustering in CR-WSNs should consider the types of nodes, as described in Section 3.2. Based on the types of nodes deployed, there are two types of hierarchical CR-WSN.

\section{(a) Hierarchical CR-WSN with All CR-WS Nodes (Hierarchical CR-WSN-all)}

This is similar to traditional hierarchical WSNs, where sensor nodes are organized into clusters, and the $\mathrm{CHs}$ serve as relays for transmitting the data. $\mathrm{CHs}$ may work for data fusion and reduce data transmission redundancy. $\mathrm{CHs}$ are selected from potential cluster head $(\mathrm{PCH})$ nodes. Figure 3 illustrates the states of CR-WS PCH nodes.

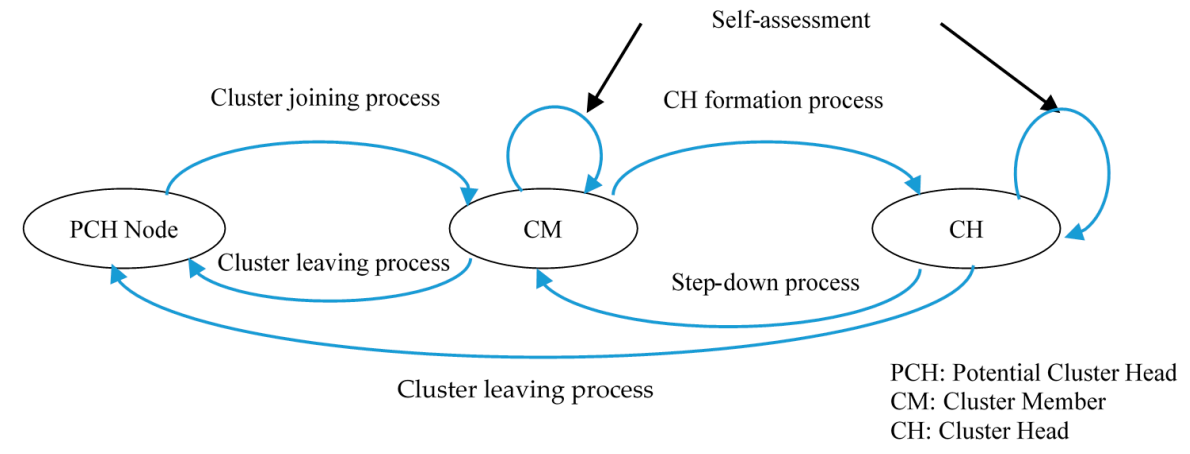

Figure 3. States of CR-WS potential cluster head $(\mathrm{PCH})$ nodes.

Although clustering algorithms and $\mathrm{CH}$-selection algorithms are similar to traditional WSNs, CR-WSNs consider one or more cognitive parameters, such as the number of idle channels available to a node, and the maximum number of nodes connected to a channel. Although deployment is easy in this topology, it is expensive in terms of energy efficiency and hardware costs.

\section{(b) Hierarchical CR-WSN with Only CHs as CR-WS Nodes (Hierarchical CR-WSN-few)}

In this type of CR-WSN, WS nodes are organized into clusters, and only CHs are equipped with CR. Other WS nodes go without CR. CHs have dedicated hardware with different transmission ranges, and are generally stationary.

$\mathrm{CHs}$ are responsible for receiving instructions from the BS and the surrounding $\mathrm{CHs}$ about the available spectrum, and they allocate channels for inter-cluster and intra-cluster communications. $\mathrm{CHs}$ gather spectrum information by sensing, and they make a channel-selection decision by selecting the best available channel for communications with CMs and the BS. CMs select and hop to the channel ordered by the $\mathrm{CHs}$ in order to switch on and start communications. Because the $\mathrm{CHs}$ have a higher transmission capacity than $\mathrm{CM}$ nodes, the minimum requirement for the number of clusters can be derived from the lower bound of the throughput.

This type of CR-WSN is more reasonable in hardware costs. Because only CHs have CR and serve without changing roles, they require better battery backup. Energy harvesting by the $\mathrm{CHs}$, and 
additional CHs for backup in every cluster, can help prolong network lifetime. In this type of network, $\mathrm{CH}$ deployment is difficult.

To the best of our knowledge, there is no study on CR-WS node-clustering algorithms that considers hierarchical CR-WSN-few along with other cognitive parameters, such as connectivity, coverage, number of CMs, spectrum availability, and, very importantly, PU protection.

A typical hierarchical network topology for a CR-WSN where only CHs are CR-WS nodes is shown in Figure 4.

Regardless of the topology used, the BS receives spectrum information from the spectrum database, its own sensing, and the CR-WS nodes.

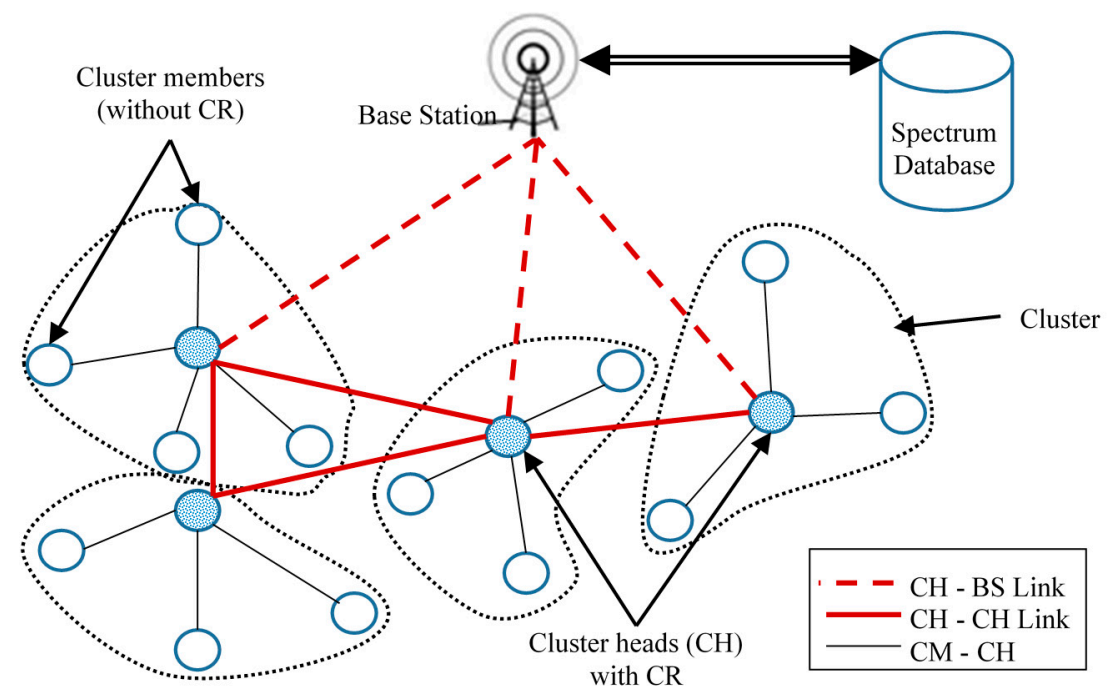

Figure 4. Hierarchical network topology for a CR-WSN where only the CHs are CR-WS nodes.

\section{Objectives and Advantages of Node Clustering in CR-WSNs}

Clustering in CR-WSNs distributes network-wide spectrum sensing and allocation issues to small groups, which solves their issues in the local area. Cluster members contribute to spectrum sensing that helps collect spectrum information from that particular area [1,19]. More information about spectrum availability reduces interference with PUs of the channels.

Clustering in CR-WSNs pursues the following objectives: (a) better local spectrum information; (b) efficient resource allocation; (c) mitigation of interference with incumbent license holders, the PUs, and cognitive users (or secondary users); (d) protect the PUs' right to access the spectrum; (e) reduce the cost of equipping all sensors with CR; (f) provide network scalability; (g) implement energy conservation; (h) reduce communications overhead; and (i) obtain better data aggregation.

The following are the advantages of clustering in large-scale and dense CR-WSNs.

\subsection{Scalability}

Scalability is the ability of a CR-WSN to adapt to its own expansion while improving efficiency. The CR-WSN is said to be scalable if it accommodates more CR-WS nodes at a later stage, after the design. Clustering in CR-WSNs efficiently deals with problems within a local cluster, and resolves issues over resource limitations. It is easy to keep localized (distributed) spectrum sensing records and update spectrum information, which is the key success factor for communications in CR-WSNs. Clustering also helps to localize the route set up within the particular geolocation, which reduces the size of the routing table stored in the individual sensor nodes. Compared with a flat topology, this kind of network topology is easier to manage, and is more scalable when responding to events in the environment. An important condition for scalability is to keep the average distance between source and destination nodes small enough as the network size grows. 


\subsection{Increased Connectivity}

Connectivity is the presence of a single- or multiple-hop path between any source and destination nodes in the CR-WSN. Network connectivity determines the efficiency of multihop routing used in CR-WSNs. Connectivity affects the network capacity, and it depends on various factors, such as interference, signal-to-noise ratio (SNR), and energy constraints [42,43]. To increase network efficiency, these issues should be solved in a local effort within the cluster.

\subsection{Licensed User Protection}

The availability of electromagnetic spectrum has a geospatial correlation. The incumbent spectrum available in one geographic location may not be available in another geographic location. Hence, it is necessary for CR-WS nodes to share spectrum information among neighbors.

Grouped CR-WS nodes deployed in the same geographic region may collect and share more information about the spectrum resource available in that particular region, which helps reduce the chances of PUs and SUs contending for the same channel at the same time. Therefore, clustering helps mitigate interference with the PUs, and protects the rights of PUs.

\subsection{Energy Conservation}

Although energy consumed for sensing and computation in WSNs is much lower than for communications, this is not the case in CR-WSNs. In CR-WSNs, CR-WS nodes have to sense and monitor channels, maintain the available-channel information, predict channel status for future use, relay the sensing report to the $\mathrm{CH}$ or BS and its neighbors, and listen to the signal of the $\mathrm{CH}, \mathrm{BS}$, and neighbor nodes for spectrum information. Therefore, CR-WS nodes have a higher duty cycle. CR-WS nodes may have multiple transceivers to work on multiple channels, especially one for a control channel and another for data channels. These transceivers must switch channels frequently; hence, they consume more energy.

Because a frequent role change for a $\mathrm{CH}$ is not always possible in CR-WSNs, as described in Section 3, preserving the energy of the $\mathrm{CH}$ as well as the CMs, is necessary.

In clustering, inter-clustering communications is performed by the cluster head. Therefore, the number of sensor nodes performing long distance communications decreases. Furthermore, as described in Section 3, clustering encourages multihop communications, which conserves energy. Therefore, clustering is an effective way to conserve energy in CR-WSNs.

\subsection{Spectrum Reuse and Bandwidth Utilization}

Multihop clustering is appropriate for large-scale CR-WSNs. In large-scale CR-WSNs, resources can be allocated orthogonally to each cluster for collision mitigation. The resources can be reused, cluster by cluster. Because clustering limits the scope of inter-cluster interactions to $\mathrm{CHs}$, and circumvents message exchange redundancy among CR-WS nodes, it can also conserve communications bandwidth.

CHs with a CR capability deployed in small clusters may collect better information about local spectrum availability. This provides opportunities to cognitive users (also known as SUs) to utilize the bandwidth, which helps achieve the CR-WSN goal to efficiently utilize spectrum unused by PUs.

\subsection{Time Synchronization}

Basically, spectrum information is gathered by cooperative sensing in CR-WSNs. In cooperative sensing, all CR-WS nodes in the network share the same quiet period, and sense the spectrum simultaneously. Time synchronization is indispensable, because a coordinated and simultaneous quiet period is used for spectrum sensing and time frame/slot allocation.

Actually, reliable signal detection is only possible by fusing sensing results from multiple sensors in the presence of shadow fading [44]. Therefore, all the clocks in a CR-WSN should be synchronized. 
Time differences can cause nodes to sense the spectrum at different times; this may lead to incorrect sensing results, and the presence of primary users may be overlooked.

Furthermore, time synchronization enables the use of time division multiple access (TDMA) techniques that are generally considered more efficient than contention-based media access control (MAC) layer techniques. Many MAC schemes for CR-WSNs may need time frame/slot structures, and require precise time synchronization for efficient operation. Some applications may also require strict time synchronization, such as tracking. Clustering can provide significant support for perfect synchronization by overcoming any inherent out-of-synchronization deficiency.

\subsection{Reducing Network Traffic by Data Aggregation and Fusion}

Data aggregation and fusion are the processes of aggregating the data from multiple sensor nodes and providing fused data to the BS. Data aggregation and fusion methods eliminate redundant transmissions, hence saving bandwidth and energy.

In densely deployed CR-WSNs, many sensors produce very similar data. Transferring similar data by all CR-WS nodes may generate network-wide massive traffic that wastes network bandwidth and other resources. In clustering, CR-WS nodes send data to the $\mathrm{CH}$ that keeps localized traffic within the cluster. Each $\mathrm{CH}$ aggregates data, and transmits the fused data to the $\mathrm{BS}$ or another $\mathrm{CH}$. Generally, $\mathrm{CHs}$ form a tree structure to transmit aggregated data via multihops through other $\mathrm{CHs}$, which results in significant energy savings [45].

\subsection{Fault-Tolerance and Robustness}

CR-WSNs are prone to failure for various reasons, such as energy depletion, hardware malfunctions, and malicious attacks. Clustering helps to apply a fault-tolerant approach locally, without affecting the complete network. Hence, the network becomes more robust, and easy to maintain and manage. Clustering provides more convenience for network topology control, and responds to channel availability changes, PU arrival, and unpredicted node failures.

\subsection{Reduced Overhead and Delay}

After clustering, not every node needs to forward data to the BS, which helps to mitigate network traffic overload, data packet collisions, and retransmissions. It also reduces frequent channel switching, and hence, reduces delay. Frequent channel switching in a CR network was described by Joshi et al. [46]. Clustering stabilizes the network topology at the sensor level, and reduces topology maintenance overhead.

Besides the applications of clustering mentioned above, clustering can be employed to perform attacker detection [47]. Because CR-WSN utilizes idle spectrum resources, it is vulnerable to selfish attack. In the attack, one or more selfish CR-WS nodes occupy spectrum that they are not using [48-50].

\section{Node Clustering in WSNs vs. CR-WSNs}

Although the objective of clustering in CR-WSNs is similar to that in WSNs (i.e., scalability, coverage, extended lifetime, robustness, better data collection, and aggregation), clustering in CR-WSNs is more challenging, because PUs are unlikely to cooperate with SUs. New cognitive radio network-related challenges are node deployment, $\mathrm{CH}$ selection, $\mathrm{PU}$ arrival prediction, channel allocation, increased complexity, error rate, and PU interference.

WSNs are generally designed for a single channel, whereas CR-WSNs require multiple channels. If any PU claims the channel currently used by CR-WS nodes, the channel must be vacated immediately within a prespecified time.

In addition to the clustering challenges faced by WSNs, CR-WSNs have to protect the right of the incumbent users to access licensed channels. The responsibilities of the $\mathrm{CHs}$ and $\mathrm{CMs}$ are different in CR-WSNs than in WSNs. It is even more difficult to handle unpredictable and frequent 
channel-status changes in CR-WSNs. Therefore, clustering schemes for WSNs cannot be directly implemented in CR-WSNs.

Trust and security should be more controlled in CR-WSNs, because some malicious or selfish nodes can intrude on the communications by interfering with the licensed channels or not changing the channel within the prespecified time period. In addition, a malicious node might report false sensing results about the channel status.

Workload for a CH in CR-WSNs is much greater than in WSNs. CHs in CR-WSNs have to focus on inter-cluster and intra-cluster communications, as well as take care of available channel information in the central spectrum database and channel availability information from neighbors and/or any other CHs. This workload is unnecessary in WSNs.

In WSNs, a basic parameter for $\mathrm{CH}$ selection is residual energy. In case of energy depletion, the $\mathrm{CH}$ responsibility switches to another node, so that a $\mathrm{CH}^{\prime}$ 's energy depletion does not break network connectivity. On the other hand, in CR-WSNs, if CHs have dedicated hardware, network lifetime depends upon the $\mathrm{CH}^{\prime}$ 's energy. Therefore, energy conservation for the $\mathrm{CH}$ is important. The active duty cycle of nodes in WSNs is very low (as low as 1\%) [51]. However, the active duty cycle is high in CR-WSNs, because CR-WS nodes have to monitor PU activities and report to the $\mathrm{CH}$.

\section{Survey of Some of the Existing Node Clustering Techniques}

Many node clustering schemes have been proposed in the literature for the different objectives and goals.

Xu et al. [10] proposed a cluster-based MAC protocol called KoN-MAC for CR-WSNs. It is based on a clustering scheme for WSNs by Lin and Gerla [52]. Pritom et al. [11] proposed a MAC protocol for cluster-based CR-WSNs, but its MAC scheme is based on the Low-Energy Adaptive Clustering Hierarchy (LEACH) protocol [53], which is specially designed for WSNs.

A novel clustering-based spectrum sensing (CBSS) in CR-WSNs was proposed by Qu et al. [12]. In this method, sensor nodes are grouped into different sets based on their similarity in sensing results. An objective function is proposed to identify the optimal cluster number. Details for cluster formation and $\mathrm{CH}$ selection were not given, and the performance was not compared with existent algorithms.

Rauniyar and Shin [13] proposed a clustering scheme for cooperative spectrum sensing based on other approaches $[20,23]$. In the scheme, a pair of nodes in a group can alternate between sleep and wake modes during the sensing process.

A spectrum-aware cluster-based energy-efficient multimedia (SCEEM) routing protocol was proposed by Shah et al. [14]. In this protocol, SU nodes form a cluster with a higher number of commonly available idle channels. The $\mathrm{CHs}$ are selected based on energy and relative spectrum awareness, such that noncontiguous available spectrum bands are clustered and scheduled to provide continuous transmission opportunities.

Salim et al. [15] proposed clustering with temporary support nodes (CENTRE). This scheme has a fixed cluster formation duration. It assumes that CMs adjust transmission power based on distance to the $\mathrm{CH}$.

Mustapha et al. [16] proposed an energy-efficient spectrum-aware reinforcement learning-based clustering (EESA-RLC) algorithm for CR-WSNs. In this algorithm, a member node learns the energy and cooperative sensing costs for neighboring clusters in order to achieve an optimal solution.

Manoor and Shahid [17] proposed a clustering scheme called BECHR for CR-WSNs. This scheme selects a $\mathrm{CH}$ based on residual energy in an iterative manner. It works like conventional WSN clustering methods but with some variations.

Eletreby et al. proposed a spectrum aware clustering protocol for CR-WSNs called CogLEACH [18]. It is an extension of the LEACH protocol, and uses the number of vacant channels as a weight in the probability for each node to become a $\mathrm{CH}$. This work evaluates $\mathrm{CogLEACH}$ in three different models. It extends the throughput and lifetime of the network, compared to the regular LEACH protocol that operates in the same settings. 
Zhang et al. [19] and Han et al. [21] proposed distributed spectrum-aware clustering (DSAC) for CR-WSNs based on group-wise constrained (GWC) agglomerative clustering (GCAC) [54]. The basic idea is to set each node as a disjoint cluster at the beginning, and to merge the two nearest clusters during each iteration until the cluster number is reduced to the optimal number. During each iteration, the inter-cluster distances are recalculated. Initially, DSAC considers each node as a $\mathrm{CH}$, and then merges $\mathrm{CHs}$ within each iteration until the number of $\mathrm{CHs}$ reaches the optimum number.

Mustapha et al. [20] proposed an energy-aware clustering (EAC) algorithm.

Ozger et al. [23] and Ozger and Akan [55] proposed event-driven spectrum-aware clustering (ESAC) and mobility-aware Event-to-sink Spectrum-Aware Clustering (mESAC) protocols that form temporal clusters for each event in CR-WSNs. These protocols determine eligible nodes for clustering based on the local position of nodes between the event and the sink. CHs are selected from among the candidate nodes based on node degree, channel availability, and distance to the sink. Because this is event-driven clustering, the clusters are immediately dismissed after finishing data transmission. Hence, it is not suitable for other scenarios.

Pei et al. [24] proposed adaptive clustering for CR-WSNs called low-energy adaptive uneven clustering hierarchy $(\mathrm{LEAUCH})$ for cognitive radio sensor networks. LEAUCH elects candidate $\mathrm{CH}$ nodes based on the number of idle channels, and selects a $\mathrm{CH}$ based on distance from the sink. To avoid the energy hole among $\mathrm{CHs}$, it employs an uneven-sized clustering method (i.e., fewer members in the clusters near the sink).

Zubair et al. [25] proposed a distributed solution to establish a common control channel in a geographic area using spatial spectral correlation for virtual clustering for CR-WSNs. It reduces route failure and energy consumption.

Nguyen and Koo [26] studied clustering for CR-WSNs. This work formulates the throughput maximization problem as mixed-integer nonlinear programming to find optimal sensor clustering and to utilize the Branch and Bound algorithm.

Shah and Akan [27] proposed a spectrum-aware cluster-based routing (SCR) protocol for CR-WSNs. Nodes are clustered based on their relative spectrum awareness and residual energy. The protocol selects $\mathrm{CHs}$ based on energy and relative spectrum awareness, such that non-contiguous available spectrum bands are clustered and scheduled to provide continuous transmission opportunities.

Park et al. [28] proposed a cognitive radio-based hybrid data-type clustering (CR-HDC) algorithm for CR-WSNs. It uses blocking and forced termination probabilities for $\mathrm{CH}$ transmission range and channel occupancy.

General objectives of clustering in CR-WSNs should include CCC establishment, stability, energy efficiency, cooperative tasks, and PU protection. Clustering metrics should be channel availability, geographic location, signal strength or channel quality, number of optimal clusters or cluster heads, etc. Many existing clustering schemes are extensions of clustering schemes for WSNs, with some performance enhancements, such as a lower number of clusters, lower clustering overhead, lower energy consumption, etc.

Table 2 summarizes the existing clustering schemes in the literature regarding their objectives, the metrics they use for clustering, performance enhancements, and $\mathrm{CH}$ selection methods.

The clustering algorithms described in Table 2 consider all nodes as being equipped with CR. Many of them have no CCC or rendezvous notion. These protocols do not consider PU protection as one of the main objectives. CHs do not have dedicated hardware, and are mostly iteratively selected based on residual energy and number of idle channels. 
Table 2. Comparison of the existing clustering schemes for CR-WSNs.

\begin{tabular}{|c|c|c|c|c|c|}
\hline Clustering Scheme & Objectives & Metrics & Performance Enhancement & CH Selection & Special Node \\
\hline KoN-MAC & MAC protocol & Similar to WSNs & Added channel weight & Elected & Gateway node \\
\hline MQ-MAC & MAC protocol & Similar to LEACH & Modified for CRNs & Predetermined probability (similar to LEACH) & Gateway node \\
\hline CBSS & $\begin{array}{l}\text { Energy efficiency } \\
\text { Cooperative task }\end{array}$ & $\begin{array}{l}\text { Similarity between } \\
\text { sensor nodes }\end{array}$ & Energy consumption & $\begin{array}{l}\text { No CH, but selects a node for } \\
\text { spectrum sensing }\end{array}$ & NA \\
\hline ECS & Cooperative spectrum sensing & $\begin{array}{l}\text { Spectrum-aware } \\
\text { node-grouping }\end{array}$ & $\begin{array}{l}\text { Energy consumption (compared with } \\
\text { DEEC algorithm [56] for WSNs }\end{array}$ & $\begin{array}{l}\text { Predetermined probability (similar to LEACH) } \\
\text { Only nodes in awake mode engage in the } \\
\text { cluster-head selection process }\end{array}$ & NA \\
\hline SCEEM & Cross-layer routing protocol & Higher spectrum rank & Optimal number of clusters & Based on highest spectrum energy rank & NA \\
\hline CENTRE & Energy efficiency & Distance to the $\mathrm{CH}$ & $\begin{array}{l}\text { Clustering overhead } \\
\text { (compared with DSAC) }\end{array}$ & Self-declared & $\begin{array}{l}\text { Temporary support } \\
\text { (TS) node }\end{array}$ \\
\hline EESA-RLC & $\begin{array}{l}\text { Energy efficient } \\
\text { PU detection }\end{array}$ & $\begin{array}{l}\text { Optimal number of } \\
\text { clusters }\end{array}$ & Cooperative probability of detection & $\begin{array}{l}\text { Number of vacant channels } \\
\text { (similar to HEED [57]) }\end{array}$ & NA \\
\hline BECHR & Spectrum sensing & NA & $\begin{array}{l}\text { Energy consumption } \\
\text { (compared with LEACH) }\end{array}$ & Iterative (based on energy level) & $\begin{array}{l}\text { No, but all nodes } \\
\text { have GPS }\end{array}$ \\
\hline CogLEACH & $\begin{array}{l}\text { Clustering evaluation in three } \\
\text { different similarity models }\end{array}$ & $\begin{array}{l}\text { Number of vacant } \\
\text { channels }\end{array}$ & Extension of LEACH & $\begin{array}{l}\text { Number of vacant channels as a weight in the } \\
\text { probability of each node becoming a } \mathrm{CH}\end{array}$ & NA \\
\hline DSAC & Energy efficiency & $\begin{array}{l}\text { Optimal number } \\
\text { of clusters }\end{array}$ & $\begin{array}{l}\text { Energy consumption } \\
\text { Scalability }\end{array}$ & $\begin{array}{c}\text { Iterative (each node has equal probability to } \\
\text { become } \mathrm{CH} \text { ) }\end{array}$ & $\begin{array}{l}\text { Only certain nodes } \\
\text { can be } \mathrm{CHs}\end{array}$ \\
\hline EAC & Energy efficiency & $\begin{array}{l}\text { Optimal number } \\
\text { of clusters }\end{array}$ & NA & $\begin{array}{l}\text { Residual energy and number of } \\
\text { neighbor nodes }\end{array}$ & NA \\
\hline ESAC/mESAC & Event-driven clustering & Spectrum availabilities & $\begin{array}{l}\text { Energy consumption } \\
\text { (with a cost of delay) }\end{array}$ & $\begin{array}{l}\text { Node degree, available channels, } \\
\text { and distance to the sink }\end{array}$ & NA \\
\hline LEAUCH & Energy efficiency & $\begin{array}{c}\text { Number of channels } \\
\text { available } \\
\text { Uneven-sized clustering }\end{array}$ & $\begin{array}{l}\text { Energy consumption } \\
\text { Network load balance }\end{array}$ & $\begin{array}{l}\text { Number of idle channels } \\
\text { Distance from the sink }\end{array}$ & NA \\
\hline Virtual clustering & $\begin{array}{l}\text { Control channel establishment } \\
\text { MAC protocol }\end{array}$ & $\begin{array}{l}\text { Similarities of spectrum } \\
\text { opportunities } \\
\text { Geographic location }\end{array}$ & $\begin{array}{l}\text { Collision mitigation } \\
\text { Control packet success rate } \\
\text { Delay }\end{array}$ & $\mathrm{NA}$ & NA \\
\hline SCR & Routing & $\begin{array}{l}\text { Spectrum measurements } \\
\text { Residual energy }\end{array}$ & $\begin{array}{l}\text { Mean packet delay } \\
\text { Energy consumption }\end{array}$ & Spectrum energy rank & NA \\
\hline CR-HDC & Energy efficiency & $\begin{array}{l}\text { Geographic location } \\
\text { Optimal transmission } \\
\text { range }\end{array}$ & NA & $\begin{array}{l}\text { Iterative (based on residual energy and } \\
\text { average one-hop distance to all other nodes) }\end{array}$ & NA \\
\hline
\end{tabular}




\section{Issues and Challenges of Node Clustering in CR-WSNs}

Along with the challenges faced by WSNs, CR-WSNs have to adapt to frequent environmental changes, such as the available bandwidth, the interference level, fading, path loss, etc. Adaptation to environmental changes includes adaptive channel coding, source coding, and modulation. It also requires using multiple channels for seamless communications. Therefore, they face more challenges than WSNs. Particularly, single point failure, proportional deployment of nodes with CR module, and backup node selection are new challenges to be addressed in CR-WSN-few. The fundamental design considerations of CR-WSNs should be as follows.

\subsection{Broadcast Support}

CR-WSNs should support broadcasting, because WS nodes need to listen for emergency signals, and leave the channel whenever incumbent license holders claim it. Additionally, some applications, such as routing protocols, use broadcast information. Many WSNs use passive sensors with an omnidirectional antenna, where all nodes communicate with each other over the same channel. Therefore, it is easy for them to support broadcast information. However, different nodes may communicate on different channels in CR-WSNs, and they may not receive broadcast information. This may cause higher delay and/or network partition.

\subsection{Busy Receiver Problem}

The busy receiver problem may occur in CR-WSNs because of the multichannel network. Transmitters cannot find their receivers on a channel because the receivers are busy on other channels (either transmitting or receiving). Thus, the busy receiver problem increases the dropped packet rate and wastes channel bandwidth.

\subsection{Control Channel Saturation Problem}

In CR-WSNs, one dedicated channel (or one dedicated control time duration) is necessary to communicate with other sensors. A problem occurs in a dense network environment when the network load increases. The control channel becomes the bottleneck preventing the data channels from being utilized efficiently. This problem is also called the control channel bottleneck problem [58].

\subsection{Multichannel Hidden Node Problem}

CR-WSNs have to cope with both the single-channel hidden node problem and the multichannel hidden node problem. To mitigate the traditional and well-known single-channel hidden node problem, request-to-send (RTS) and clear-to-send (CTS) handshakes are used. However, if nodes utilize multiple channels, a node may miss channel negotiation packets, because they are busy communicating on another channel, and the multichannel hidden node problem occurs. Details of the multichannel hidden node problem can be found elsewhere $[59,60]$.

\subsection{Fault-Tolerance}

In order to avoid the loss of significant data from nodes, fault-tolerant $\mathrm{CHs}$ are usually required. Re-clustering is considered the most intuitive method to recover from a cluster failure. However, re-clustering is difficult in CR-WSNs, especially if only the $\mathrm{CH}$ has dedicated hardware. Deploying multiple $\mathrm{CHs}$ for backup is a viable solution for $\mathrm{CH}$ failure.

\subsection{Energy Hole Avoidance}

In a large-scale CR-WSN, CR-WS nodes forward packets in multihop fashion to deliver the collected data to a BS. The nodes closer to the BS have to receive and retransmit more packets generated by the sensors than those farther away from the BS. This leads to energy depletion in the nodes closer to the BS, leaving a hole near the BS called an energy hole. This phenomenon partitions 
the whole network, and prevents outside nodes from sending information to the BS, even though many nodes in CR-WSNs still have plenty of energy. It is difficult to solve the energy hole problem completely; however, it can be mitigated by efficient node deployment, energy harvesting, and efficient $\mathrm{CH}$ selection strategies.

\subsection{Single Point of Failure}

With CR-WSN-few, the $\mathrm{CH}$ has special hardware and is relatively more vulnerable to $\mathrm{CH}$ failure (also called the single point of failure) than in a CR-WSN-all.

\subsection{Node Deployment}

Clustering in CR-WSNs brings several deployment challenges, such as ensuring PU access rights, SU connectivity, selection of the optimal channel, $\mathrm{CH}$ selection (under CR-WSN-all), CH deployment (under CR-WSN-few), and optimal cluster sizes. For measuring an accurate degree of optimum coverage, sensing PU activities, and maintaining connectivity, an optimal strategy for node deployment is essential. Node deployment could be different based on the objectives, such as: (1) PU protection; (2) coverage and spectrum information collection; (3) minimal energy consumption configuration; (4) reduction of event-sensing delay; and (5) reduction of the control channel saturation problem.

\subsection{Optimal Number of Clusters}

One of the challenges in cluster-based CR-WSNs is to determine the optimal number of clusters. A large number of clusters leads to establishing long routes, which naturally incur higher delay. On the other hand, a small number of clusters exhausts the energy of the $\mathrm{CH}$, and incurs inefficient spectrum sharing.

The cluster establishment procedure and its complexity should be independent of the network scale, and should remain constant. A fixed cluster size has load-balancing and energy-consumption issues. In particular, $\mathrm{CHs}$ that are closer to the BS need to forward more inter-cluster packets, and hence, will deplete their battery power sooner than the CHs that are farther away from the BS. Forming relatively small clusters near the BS distributes the workload among multiple CHs.

When CHs are specialized CR-WS nodes (under CR-WSN-few), there should be a CR-WSN with fewer $\mathrm{CHs}$, because the $\mathrm{CH}$ s tend to be more expensive and bigger in size than the CMs. These nodes can be easily visible and detectable if deployed for critical application fields, such as battlefields, border security, infrastructure security, etc.

\subsection{Connectivity}

Connectivity is one major goal of node clustering in CR-WSNs. Ideally, a CM is able to communicate with its $\mathrm{CH}$, either in one hop or in multiple hops, in intra-cluster communications. In inter-cluster communications, nodes can communicate via gateway nodes or via $\mathrm{CHs}$.

\subsection{Node Synchronization}

Synchronization ensures CR-WS node activities start simultaneously in the whole network. Therefore, in distributed clustering algorithms, node synchronization is very important for better performance. Lack of synchronization may result in a suboptimal choice of data channels and may interfere with PU activities. Although some synchronization-based clustering methods for CR-WSNs exist in the literature $[14,18]$, getting perfect synchronization is difficult.

Detailed challenges of CR-WSNs are well studied in [1].

\section{Conclusions and Future Direction}

In large-scale CR-WSNs, clustering is an effective way to achieve goals like obtaining information on local spectrum, efficient resource allocation, interference mitigation, $\mathrm{PU}$ protection, coverage and 
deployment cost reduction, network scalability, communications overhead reduction, an extended lifetime, robustness, and better data collection and aggregation. Clustering is very important in CR-WSNs because it provides a topology control approach to reduce transmission overhead and to exploit data aggregation among a large number of CR-WS nodes. Clustering in CR-WSNs is different from conventional clustering algorithms in WSNs in various ways. CR-WS nodes in CR-WSNs are responsible for channel sensing, channel allocation, data collection and data aggregation, including other cognitive tasks. Cluster heads in clustering help data aggregation and fusion, which intensely reduces the number of nodes taking part in packet transmission, and prolongs the overall network lifetime.

In a small indoor or a favorable area, where the BS is very close to the sensors (typically, just one hop), a flat CR-WSN can be advantageous. That is because the BS is at least two hops away after clustering. Clustering also increases cluster formation and maintenance overhead.

One critical step in clustering is to deploy the CHs uniformly to conserve energy, and mitigate interference with the PU of the channel. If cost, size, and energy are not the issue, most existing clustering algorithms for WSNs, which are primarily focused on scalability and energy conservation, can be tailored to CR-WSNs. The primary focus of the algorithm should be protection of the PU's right to access the channel. Otherwise, designing CR-WSNs with only the $\mathrm{CH}$ having a CR module can be the best way.

Clustering in CR-WSNs brings several unique challenges to deployment, such as mitigating interference with PUs, ensuring connectivity among WS nodes, determining optimal cluster sizes, etc. Clustering in CR-WSNs is still in its infancy, and several key issues are yet to be addressed, such as interference mitigation, the optimal number of $\mathrm{CHs}$ and backup $\mathrm{CHs}$, cooperative sensing with and without clustering, among others. Although the optimum number of clusters in WSNs has been addressed by many researchers, to the best of our knowledge, there is no such work on CR-WSNs.

We provide the impetus required for further research to solve energy conservation, load balancing, PU protection, and efficient bandwidth utilization in CR-WSNs.

Acknowledgments: This research was supported in part by Basic Science Research Program through the National Research Foundation of Korea (NRF) funded by the Ministry of Education (NRF-2015R1D1A1A01058751) and in part by the MSIP (Ministry of Science, ICT and Future Planning), Korea, under the ITRC (Information Technology Research Center) support program (IITP-2016-R2718-16-0035) supervised by the IITP (National IT Industry Promotion Agency).

Author Contributions: Both authors wrote the paper and read and approved the final manuscript.

Conflicts of Interest: The authors declare no conflict of interest.

\section{References}

1. Joshi, G.P.; Nam, S.Y.; Kim, S.W. Cognitive radio wireless sensor networks: Applications, challenges and research trends. Sensors 2013, 13, 11196-11228. [CrossRef] [PubMed]

2. Nourmand, R. Cognitive Radio Wireless Sensor Networks. Available online: https://rezanourmand. wordpress.com/2015/04/10/271/ (accessed on 20 July 2016).

3. Ding, G.; Wang, J.; Wu, Q.; Song, F.; Chen, Y. Spectrum Sensing in Opportunity-Heterogeneous Cognitive Sensor Networks: How to Cooperate? IEEE Sens. J. 2013, 13, 4247-4255. [CrossRef]

4. Akan, O.; Karli, O.; Ergul, O. Cognitive radio sensor networks. IEEE Netw. 2009, 23, 34-40. [CrossRef]

5. Yu, J.Y.; Chong, P.H.J. A survey of clustering schemes for mobile ad hoc networks. IEEE Commun. Surv. Tutor. 2005, 7, 32-48. [CrossRef]

6. Zhang, C.; Hou, E.; Ansari, N. Node Clustering. In A Networking Perspective; John Wiley \& Sons, Inc.: Hoboken, NJ, USA, 2009; p. 173.

7. Abbasi, A.A.; Younis, M. A survey on clustering algorithms for wireless sensor networks. Comput. Commun. 2007, 30, 2826-2841. [CrossRef]

8. Yau, K.L.A.; Ramli, N.; Hashim, W.; Mohamad, H. Clustering algorithms for cognitive radio networks: A survey. J. Netw. Comput. Appl. 2014, 45, 79-95. [CrossRef] 
9. Wang, L.; Wang, J.; Ding, G.; Song, F.; Wu, Q. A survey of cluster-based cooperative spectrum sensing in cognitive radio networks. In Proceedings of the IEEE Cross Strait Quad-Regional Radio Science and Wireless Technology Conference, Harbin, China, 26-30 July 2011; Volume 1, pp. 247-251.

10. Xu, Y.; Wu, C.; He, C.; Jiang, L. A cluster-based energy efficient MAC protocol for multi-hop cognitive radio sensor networks. In Proceedings of the IEEE Global Communications Conference, Anaheim, CA, USA, 3-7 December 2012; pp. 537-542.

11. Pritom, M.M.A.; Sarker, S.; Razzaque, M.A.; Hassan, M.M.; Hossain, M.A.; Alelaiwi, A. A multiconstrained QoS aware MAC protocol for cluster-based cognitive radio sensor networks. Int. J. Distrib. Sens. Netw. 2015, 2015. [CrossRef]

12. Qu, Z.; Xu, Y.; Yin, S. A novel clustering-based spectrum sensing in cognitive radio wireless sensor networks. In Proceedings of the IEEE 3rd International Conference on Cloud Computing and Intelligence Systems, Shenzhen, China, 27-29 November 2014; pp. 695-699.

13. Rauniyar, A.; Shin, S.Y. A novel energy-efficient clustering based cooperative spectrum sensing for cognitive radio sensor networks. Int. J. Distrib. Sens. Netw. 2015, 2015. [CrossRef]

14. Shah, G.A.; Alagoz, F.; Fadel, E.A.; Akan, O.B. A spectrum-aware clustering for efficient multimedia routing in cognitive radio sensor networks. IEEE Trans. Veh. Technol. 2014, 63, 3369-3380. [CrossRef]

15. Salim, S.; Moh, S.; Choi, D.; Chung, I. An Energy-Efficient and Compact Clustering Scheme with Temporary Support Nodes for Cognitive Radio Sensor Networks. Sensors 2014, 14, 14634-14653. [CrossRef] [PubMed]

16. Mustapha, I.; Ali, B.M.; Rasid, M.F.A.; Sali, A.; Mohamad, H. An Energy-Efficient Spectrum-Aware Reinforcement Learning-Based Clustering Algorithm for Cognitive Radio Sensor Networks. Sensors 2015, 15, 19783-19818. [CrossRef] [PubMed]

17. Mansoor, U.; Shahid, M.K. Cluster based Energy Efficient Sensing for Cognitive Radio Sensor Networks. Int. J. Comput. Appl. 2014, 88, 14-19. [CrossRef]

18. Eletreby, R.M.; Elsayed, H.M.; Khairy, M.M. CogLEACH: A spectrum aware clustering protocol for cognitive radio sensor networks. In Proceedings of the IEEE 9th International Conference on Cognitive Radio Oriented Wireless Networks and Communications, Oulu, Finland, 2-4 June 2014; pp. 179-184.

19. Zhang, H.; Zhang, Z.; Dai, H.; Yin, R.; Chen, X. Distributed spectrum-aware clustering in cognitive radio sensor networks. In Proceedings of the IEEE Global Telecommunications Conference, Houston, TX, USA, 5-9 December 2011; pp. 1-6.

20. Mustapha, I.; Ali, B.M.; Sali, A.; Rasid, M.F.; Mohamad, H. Energy-aware cluster based cooperative spectrum sensing for cognitive radio sensor networks. In Proceedings of the IEEE 2nd International Symposium on Telecommunication Technologies, Langkawi, Malaysia, 24-26 November 2014; pp. 45-50.

21. Han, J.; Jeon, W.S.; Jeong, D.G. Energy-efficient channel management scheme for cognitive radio sensor networks. IEEE Trans. Veh. Technol. 2011, 60, 1905-1910. [CrossRef]

22. Zhang, H.; Zhang, Z.; Yuen, C. Energy-efficient spectrum-aware clustering for cognitive radio sensor networks. Chin. Sci. Bull. 2012, 57, 3731-3739. [CrossRef]

23. Ozger, M.; Fadel, E.; Akan, O. Event-to-sink Spectrum-Aware Clustering in Mobile Cognitive Radio Sensor Networks. IEEE Trans. Mob. Comput. 2015, 15, 2221-2233. [CrossRef]

24. Pei, E.; Han, H.; Sun, Z.; Shen, B.; Zhang, T. LEAUCH: Low-energy adaptive uneven clustering hierarchy for cognitive radio sensor network. EURASIP J. Wirel. Commun. Netw. 2015, 2015, 1-8. [CrossRef]

25. Zubair, S.; Fisal, N.; Abazeed, M.B.; Salihu, B.A.; Khan, A.S. Lightweight distributed geographical: A lightweight distributed protocol for virtual clustering in geographical forwarding cognitive radio sensor networks. Int. J. Commun. Syst. 2015, 28, 1-18. [CrossRef]

26. Nguyen, T.T.; Koo, I. Sensor Clustering and Sensing Technology for Optimal Throughput of Sensor-Aided Cognitive Radio Networks Supporting Multiple Licensed Channels. Int. J. Distrib. Sens. Netw. 2015, 2015, 1-10. [CrossRef]

27. Shah, G.A.; Akan, O.B. Spectrum-aware cluster-based routing for cognitive radio sensor networks. In Proceedings of the IEEE International Conference on Communications, Budapest, Hungary, 9-13 June 2013; pp. 2885-2889.

28. Park, J.H.; Nam, Y.; Chung, J.M. Sustainability Enhancement Multihop Clustering in Cognitive Radio Sensor Networks. Int. J. Distrib. Sens. Netw. 2015, 2015, 1-8. [CrossRef]

29. Zhao, F.; Liu, J.; Liu, J.; Guibas, L.; Reich, J. Collaborative signal and information processing: An information-directed approach. Proc. IEEE 2003, 91, 1199-1209. [CrossRef] 
30. Sohrabi, K.; Manriquez, B.; Pottie, G.J. Near ground wideband channel measurement in 800-1000 MHz. In Proceedings of the IEEE 49th Vehicular Technology Conference, Houston, TX, USA, 16-20 May 1999; Volume 1, pp. 571-574.

31. Arunraja, M.; Malathi, V.; Sakthivel, E. Energy conservation in WSN through multilevel data reduction scheme. Microprocess. Microsyst. 2015, 39, 348-357. [CrossRef]

32. Marinkovic, S.J.; Popovici, E.M.; Spagnol, C.; Faul, S.; Marnane, W.P. Energy-Efficient Low Duty Cycle MAC Protocol for Wireless Body Area Networks. IEEE Trans. Inf. Technol. Biomed. 2009, 13, 915-925. [CrossRef] [PubMed]

33. Liu, X. A survey on clustering routing protocols in wireless sensor networks. Sensors 2012, 12, 11113-11153. [CrossRef] [PubMed]

34. Soua, R.; Minet, P. A survey on energy efficient techniques in wireless sensor networks. In Proceedings of the IEEE 4th Joint IFIP Wireless and Mobile Networking Conference, Toulouse, France, 26-28 October 2011; pp. 1-9.

35. Gilbert, J.M.; Balouchi, F. Comparison of energy harvesting systems for wireless sensor networks. Int. J. Autom. Comput. 2008, 5, 334-347. [CrossRef]

36. Seah, W.K.; Eu, Z.A.; Tan, H.P. Wireless sensor networks powered by ambient energy harvesting (WSN-HEAP)-Survey and challenges. In Proceedings of the IEEE 1st International Conference on Wireless Communication, Vehicular Technology, Information Theory and Aerospace \& Electronic Systems Technology, Aalborg, Denmark, 17-20 May 2009; pp. 1-5.

37. Zheng, J.; Jamalipour, A. Wireless Sensor Networks: A Networking Perspective; John Wiley \& Sons: Hoboken, NJ, USA, 2009.

38. Younis, O.; Krunz, M.; Ramasubramanian, S. Node clustering in wireless sensor networks: Recent developments and deployment challenges. IEEE Netw. 2006, 20, 20-25. [CrossRef]

39. Kumarawadu, P.; Dechene, D.J.; Luccini, M.; Sauer, A. Algorithms for node clustering in wireless sensor networks: A survey. In Proceedings of the IEEE 4th International Conference on Information and Automation for Sustainability, Colombo, Sri Lanka, 12-14 December 2008; pp. 295-300.

40. Boyinbode, O.; Le, H.; Takizawa, M. A survey on clustering algorithms for wireless sensor networks. Int. J. Space-Based Situat. Comput. 2011, 1, 130-136. [CrossRef]

41. Mamalis, B.; Gavalas, D.; Konstantopoulos, C.; Pantziou, G. Clustering in wireless sensor networks. In RFID and Sensor Networks: Architectures, Protocols, Security and Integrations; Zhang, Y., Yang, L.T., Chen, J., Eds.; CRC Press: Boca Raton, FL, USA, 2009; pp. 324-353.

42. Ramanathan, R.; Rosales-Hain, R. Topology control of multihop wireless networks using transmit power adjustment. In Proceedings of the IEEE Nineteenth Annual Joint Conference of the IEEE Computer and Communications Societies, Tel Aviv, Israel, 26-30 March 2000; Volume 2, pp. 404-413.

43. Watts, D.J.; Strogatz, S.H. Collective dynamics of "small-world" networks. Nature 1998, 393, 440-442. [CrossRef] [PubMed]

44. Ruttik, K.; Koufos, K.; Jäntti, R. Distributed power detection in shadowing environment and with communication constraint. In Proceedings of the IEEE 18th International Symposium on Personal, Indoor and Mobile Radio Communications, Athens, Greece, 3-7 September 2007; pp. 1-5.

45. Ozdemir, S.; Xiao, Y. Secure data aggregation in wireless sensor networks: A comprehensive overview. Comput. Netw. 2009, 53, 2022-2037. [CrossRef]

46. Joshi, G.P.; Kim, S.W.; Nam, S.Y. Routing Layer Solution for Mitigating Frequent Channel Switching in Ad Hoc Cognitive Radio Networks. IEEE Commun. Lett. 2015, 19, 1917-1920. [CrossRef]

47. Ding, G.; Wu, Q.; Yao, Y.D.; Wang, J.; Chen, Y. Kernel-Based Learning for Statistical Signal Processing in Cognitive Radio Networks: Theoretical Foundations, Example Applications, and Future Directions. IEEE Signal Process. Mag. 2013, 30, 126-136. [CrossRef]

48. Jo, M.; Han, L.; Kim, D.; In, H.P. Selfish attacks and detection in cognitive radio Ad-Hoc networks. IEEE Netw. 2013, 27, 46-50.

49. Feng, J.; Zhang, Y.; Lu, G.; Zhang, L. Securing Cooperative Spectrum Sensing against Rational SSDF Attack in Cognitive Radio Networks. KSII Trans. Int. Inf. Syst. 2014, 8, 1-17.

50. Liu, Q.; Gao, J.; Guo, Y.; Liu, S. Attack-Proof Cooperative Spectrum Sensing Based on Consensus Algorithm in Cognitive Radio Networks. KSII Trans. Int. Inf. Syst. 2010, 4, 1042-1062. [CrossRef] 
51. Polastre, J.; Hill, J.; Culler, D. Versatile low power media access for wireless sensor networks. In Proceedings of the 2nd International Conference on Embedded Networked Sensor Systems, Baltimore, MD, USA, 3-5 November 2004; pp. 95-107.

52. Lin, C.R.; Gerla, M. A distributed architecture for multimedia in dynamic wireless networks. In Proceedings of the IEEE Global Telecommunications Conference, Singapore, 14-16 November 1995; Volume 2, pp. 1468-1472.

53. Heinzelman, W.R.; Chandrakasan, A.; Balakrishnan, H. Energy-efficient communication protocol for wireless microsensor networks. In Proceedings of the 33rd Annual Hawaii International Conference on System Sciences, Maui, HI, USA, 4-7 January 2000; pp. 1-10.

54. Klein, D.; Kamvar, S.D.; Manning, C.D. From Instance-Level Constraints to Space-Level Constraints: Making the Most of Prior Knowledge in Data Clustering; Technical Report; Stanford University: Stanford, CA, USA, 2002.

55. Ozger, M.; Akan, O.B. Event-driven spectrum-aware clustering in cognitive radio sensor networks. In Proceedings of the IEEE INFOCOM, Turin, Italy, 14-19 April 2013; pp. 1483-1491.

56. Qing, L.; Zhu, Q.; Wang, M. Design of a distributed energy-efficient clustering algorithm for heterogeneous wireless sensor networks. Comput. Commun. 2006, 29, 2230-2237. [CrossRef]

57. Younis, O.; Fahmy, S. HEED: A hybrid, energy-efficient, distributed clustering approach for ad hoc sensor networks. IEEE Trans. Mob. Comput. 2004, 3, 366-379. [CrossRef]

58. Joshi, G.P.; Kim, S.W. Mitigating the control channel bottleneck problem in dense cognitive radio networks. Int. J. Phys. Sci. 2011, 6, 4832-4837.

59. So, J.; Vaidya, N.H. Multi-channel MAC for ad hoc networks: Handling multi-channel hidden terminals using a single transceiver. In Proceedings of the 5th ACM International Symposium on Mobile Ad Hoc Networking and Computing, Tokyo, Japan, 24-26 May 2004; pp. 222-233.

60. Almotairi, K.H.; Shen, X.S. Multichannel medium access control for ad hoc wireless networks. Wirel. Commun. Mob. Comput. 2013, 13, 1047-1059. [CrossRef]

(C) 2016 by the authors; licensee MDPI, Basel, Switzerland. This article is an open access article distributed under the terms and conditions of the Creative Commons Attribution (CC-BY) license (http://creativecommons.org/licenses/by/4.0/). 